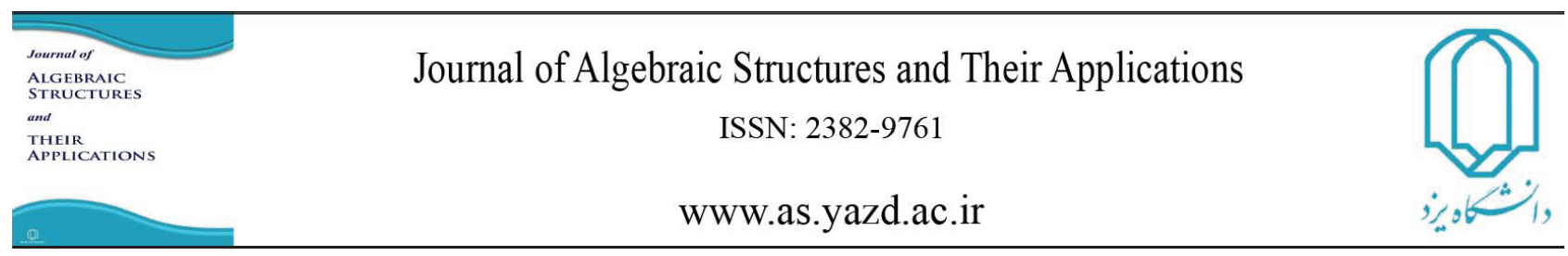

Algebraic Structures and Their Applications Vol. 5 No. 2 (2018 ) pp 1-13.

\title{
ON QUASI-ZERO DIVISOR GRAPHS OF NON-COMMUTATIVE RINGS
}

\author{
RAZIEH AMIRJAN AND EBRAHIM HASHEMI
}

Communicated by S. Alikhani

\begin{abstract}
Let $R$ be an associative ring with identity. A ring $R$ is called reversible if $a b=0$, then $b a=0$ for $a, b \in R$. The quasi-zero-divisor graph of $R$, denoted by $\Gamma^{*}(R)$ is an undirected graph with all nonzero zero-divisors of $R$ as vertex set and two distinct vertices $x$ and $y$ are adjacent if and only if there exists $0 \neq r \in R \backslash(\operatorname{ann}(x) \cup \operatorname{ann}(y))$ such that $x r y=0$ or $y r x=0$. In this paper, we determine the diameter and girth of $\Gamma^{*}(R)$. We show that the zero-divisor graph of $R$ denoted by $\Gamma(R)$, is a spanning subgraph of $\Gamma^{*}(R)$. Also, we investigate when $\Gamma^{*}(R)$ is identical to $\Gamma(R)$. Moreover, for a reversible ring $R$, we study the diameter and girth of $\Gamma^{*}(R[x])$ and we investigate when $\Gamma^{*}(R[x])$ is identical to $\Gamma(R[x])$.
\end{abstract}

\section{INTRODUCTION}

One of the interesting and active area in the last decade is using graph theoretical tools to study the algebraic structures. There are several papers devoted to study of rings in this approach, see for example [T], [2], [3] and [4].

DOI:http://dx.doi.org/10.29252/asta.5.2.1

MSC(2010): 16U99, 05C99

Keywords: quasi-zero-divisor graph, zero-divisor graph, reversible ring, reduced ring, diameter.

Received: 14 July 2018, Accepted: 19 October 2018

$*$ Corresponding author

(C) 2018 Yazd University. 
Throughout this paper, $R$ denotes an associative ring with identity. For an element $a \in R$, let $\ell \cdot \operatorname{ann}(a)=\ell_{R}(a)=\{b \in R \mid b a=0\}$ the left annihilator of an element $a \in R$ and the right annihilator of $a$ denoted by $\operatorname{rann}(a)=r_{R}(a)$, is the set $\{b \in R \mid a b=0\}$. Also $\operatorname{ann}(x)=\mathrm{r} \cdot \operatorname{ann}(x) \cup \ell \cdot \operatorname{ann}(x)$. We write $Z_{\ell}(R), Z_{r}(R)$ and $Z(R)$ the set of all left zero-divisors of $R$, the set of right zero-divisors of $R$, and the $Z_{\ell}(R) \cup Z_{r}(R)$, respectively. We use $\operatorname{Min}(R)$ to denote the set of all minimal prime ideals and $\operatorname{Nil}(R)$ to denote the set of all nilpotent elements of $R$. For any $A \subseteq R$, let $A^{*}=A \backslash\{0\}$. A ring $R$ is said to be reduced if $\operatorname{Nil}(R)=0$, and a ring $R$ is said to be reversible if $a b=0$ implies that $b a=0$ for each $a, b \in R$. Clearly commutative rings and reduced rings are reversible. All other terminology is standard, and definitions can be found in [110].

Let $\Gamma=(V, E)$ be a graph, where $V=V(\Gamma)$ is the set of vertices and $E=E(\Gamma)$ is the set of edges. A graph is said to be connected if for each pair of distinct vertices $v$ and $w$, there is a finite sequence of distinct vertices $v_{1}=v, v_{2}, \ldots, v_{n}=w$ such that each pair $\left\{v_{i}, v_{i+1}\right\}$ is an edge, such a sequence is said to be a path and the distance, $d_{\Gamma}(v, w)$, between connected vertices $v$ and $w$ is the length of the shortest path connecting them. The diameter of a connected graph $\Gamma$, denoted by $\operatorname{diam}(\Gamma)$, is the maximum distance between any pair of the vertices of $\Gamma$. The girth of a graph $\Gamma$, denoted by $g(\Gamma)$, is the length of the shortest cycle in $\Gamma$. A graph with no cycle has infinite girth. We denote the complete graph with $n$ vertices by $K^{n}$ and we denote the complete bipartite graph by $K^{m, n}$. For two vertices $u$ and $v$ in $\Gamma$, the notation $u-v$ means that $u$ and $v$ are adjacent. For two arbitrary graphs $\Gamma$ and $\Gamma^{*}$, If $\Gamma$ is identical to $\Gamma^{*}$, then we write $\Gamma=\Gamma^{*}$; otherwise, we write $\Gamma \neq \Gamma^{*}$. Any undefined notation or terminology is standard, as in [14].

The concept of a zero-divisor graph of a commutative ring $R$ was introduced by Beck in [4]. However, he let all elements of $R$ be vertices of the graph and was mainly interested in coloring. Inspired by his study, Anderson and Livingston [I], refined and studied the (undirected) zerodivisor graph $\Gamma(R)$, whose vertices are the nonzero zero-divisors of a ring such that distinct vertices $x$ and $y$ are adjacent in $\Gamma(R)$ if and only if $x y=0$. The relationship between ringtheoretic properties of commutative ring $R$ and graph theoretic properties of $\Gamma(R)$ has been studied extensively. For example, $\Gamma(R)$ is connected with $\operatorname{diam}(\Gamma(R)) \leq 3$, and $\mathrm{g}(\Gamma(R)) \leq 4$ if $\Gamma(R)$ contains a cycle [I, Theorems 2.3 and 2.4].

If $R$ is a non-commutative ring, Redmond [1:3], defined a directed zero-divisor graph in a similar way. A directed graph is connected if there exists a directed path connecting any two distinct vertices. The distance and the diameter are defined in a similar way as well, having in mind that all paths in question are directed. Redmond, also defined an undirected zero-divisor graph of a non-commutative ring $R$, the graph $\Gamma(R)$, with vertices in the set $Z(R)^{*}$ and such 
that for distinct vertices $a$ and $b$ there is an edge connecting them if and only if $a b=0$ or $b a=0$.

For a commutative ring $R$, Badawi [3], defined the concept of the annihilator graph of $R$, denoted by $A G(R)$, which is an undirected graph with vertex set $Z(R)^{*}$ and two distinct vertices $x$ and $y$ are adjacent if and only if $\operatorname{ann}(x y) \neq \operatorname{ann}(x) \cup \operatorname{ann}(y)$. He showed that $A G(R)$ is connected and $\operatorname{diam}(A G(R)) \leq 2$ and $\mathrm{g}(A G(R)) \leq 4$ if $A G(R)$ contains a cycle.

Zhao et al. [15], introduced the quasi-zero-divisor graph of a ring $R$, denoted by $\Gamma_{*}(R)$, which is a directed graph defined on its nonzero quasi-zero-divisors, where $x \rightarrow y$ is an edge between disjoint vertices $x$ and $y$ if and only if $x R y=0$. An element $a \in R$ is said to be a left quasi-zero-divisor (resp., right quasi-zero-divisor) if there exists $0 \neq b \in R$ such that $a R b=0$ (resp., $b R a=0$ ). An element in $R$ is called a quasi-zero-divisor of $R$ if it is a left or right quasi-zero-divisor. They studied $\Gamma_{*}(R)$, when the base ring $R$, is semiprime and FIC. A ring $R$ is said to be semiprime if it has no nonzero nilpotent ideal, or equivalently, the intersection of minimal prime ideals of $R$ is zero. A ring $\mathrm{R}$ is called FIC, if it satisfies the following condition: for any finite ideal $I$ of $R, \Gamma_{*}(R / I)$ having an infinite clique implies that $\Gamma_{*}(R)$ contains an infinite clique. They also defined the undirected quasi-zero-divisor graph of $R$, denoted by $\bar{\Gamma}_{*}(R)$ on nonzero quasi-zero-divisors, such that two distinct vertices $x$ and $y$ are adjacent if and only if $x R y=0$ or $y R x=0$. They showed that $\bar{\Gamma}_{*}(R)$ is connected, $\operatorname{diam}\left(\bar{\Gamma}_{*}(R)\right) \leq 3$ and if $R$ contains a cycle, then $g\left(\bar{\Gamma}_{*}(R)\right) \leq 4$. The graphs $\Gamma_{*}(R)$ and $\bar{\Gamma}_{*}(R)$ are subgraphs of $\Gamma(R)$.

In this paper, we introduce the quasi-zero-divisor graph for a non-commutative ring $R$. The quasi-zero-divisor graph of $R$, denoted by $\Gamma^{*}(R)$ is an undirected graph with vertex set $Z(R)^{*}$, and two distinct vertices $x$ and $y$ are adjacent if and only if there exists $0 \neq r \in$ $R \backslash(\operatorname{ann}(x) \cup \operatorname{ann}(y))$ such that $x r y=0$ or $y r x=0$. If $R$ is a commutative ring, then $\Gamma^{*}(R)=A G(R)$. Thus $\Gamma^{*}(R)$ is a generalization of $A G(R)$. We show that $\Gamma(R)$ is a spanning subgraph of $\Gamma^{*}(R)$, and we determine the diameter and girth of $\Gamma^{*}(R)$. Also, we investigate when $\Gamma^{*}(R)$ is identical to $\Gamma(R)$. Moreover, for a reversible ring $R$, we study the diameter and girth of $\Gamma^{*}(R[x])$ and we investigate when $\Gamma^{*}(R[x])$ is identical to $\Gamma(R[x])$.

\section{Quasi-Zero-Divisor Graphs of Reversible Rings}

In this section, at first we study some basic properties of $\Gamma^{*}(R)$. Then we determine the diameter and girth of $\Gamma^{*}(R)$, when $R$ is reversible. We also investigate when $\Gamma^{*}(R)=\Gamma(R)$. We start this section with the following definition.

Definition 2.1. Let $R$ be an associative ring with identity. The quasi-zero-divisor graph of $R$, denoted by $\Gamma^{*}(R)$, is an undirected graph with all nonzero zero-divisors of $R$ as vertex set and two distinct vertices $x$ and $y$ are adjacent if and only if there exists $0 \neq r \in R \backslash(\operatorname{ann}(x) \cup \operatorname{ann}(y))$ such that $x r y=0$ or $y r x=0$. 
Let $R$ be an associative ring with identity and let $x$ and $y$ be distinct elements of $Z(R)^{*}$. If $x$ and $y$ are adjacent in $\Gamma(R)$, then $x 1_{R} y=0$ or $y 1_{R} x=0$ and so $x$ and $y$ are adjacent in $\Gamma^{*}(R)$. In particular, each path in $\Gamma(R)$ is a path in $\Gamma^{*}(R)$. So the zero-divisor graph $\Gamma(R)$ is a spanning subgraph of $\Gamma^{*}(R)$. Thus we have the following result.

Theorem 2.2. Let $R$ be an associative ring with identity. Then $\Gamma^{*}(R)$ is connected, $\operatorname{diam}\left(\Gamma^{*}(R)\right) \leq 3$, and $\mathrm{g}\left(\Gamma^{*}(R)\right) \leq 4$ if $\Gamma^{*}(R)$ contains a cycle.

Proof. Since $V(\Gamma(R))=V\left(\Gamma^{*}(R)\right)=Z(R)^{*}$ and $\Gamma(R)$ is a spanning subgraph of $\Gamma^{*}(R)$, by [133, Theorem 3.2], $\Gamma(R)$ is connected and $\operatorname{diam}(\Gamma(R)) \leq 3$. By [133, Theorem 3.3], $\mathrm{g}(\Gamma(R)) \leq 4$ if $\Gamma(R)$ contains a cycle. Thus $\Gamma^{*}(R)$ is connected, $\operatorname{diam}\left(\Gamma^{*}(R)\right) \leq 3$. Moreover, $\mathrm{g}\left(\Gamma^{*}(R)\right) \leq 4$ if $\Gamma^{*}(R)$ contains a cycle.

In the following lemma we show that for a reversible ring $R, \operatorname{diam}\left(\Gamma^{*}(R)\right) \leq 2$.

Lemma 2.3. Let $R$ be a reversible ring. If $x$ and $y$ are distinct elements of $Z(R)^{*}$ and $d_{\Gamma(R)}(x, y)=3$, then $d_{\Gamma^{*}(R)}(x, y)=1$, and therefore $\operatorname{diam}\left(\Gamma^{*}(R)\right) \leq 2$.

Proof. Let $x$ and $y$ be distinct elements of $Z(R)^{*}$. If $d_{\Gamma(R)}(x, y)=3$, then $x y \neq 0$ and $x, y$ have no mutual annihilator, and there exist distinct elements $a, b \in Z(R)^{*} \backslash\{x, y\}$ such that $a x=0, y b=0, y a \neq 0, b x \neq 0$. We consider $r=a+b$, if $r=0$, then $a=-b$, a contradiction. On the other hand, $r \in R \backslash(\operatorname{ann}(x) \cup \operatorname{ann}(y))$ and $x(a+b) y=0$. So $d_{\Gamma^{*}(R)}(x, y)=1$. Thus $\operatorname{diam}\left(\Gamma^{*}(R)\right) \leq 2$.

In the next theorem we give a necessary condition for $\mathrm{g}\left(\Gamma^{*}(R)\right)=3$, when $R$ is a reversible ring and $\Gamma^{*}(R) \neq \Gamma(R)$.

Theorem 2.4. Let $R$ be a reversible ring. Suppose that $x$ and $y$ are distinct elements of $Z(R)^{*}$ such that $x-y$ is an edge of $\Gamma^{*}(R)$ that is not an edge of $\Gamma(R)$. If $x y^{2} \neq 0$ and $x^{2} y \neq 0$, then there exists $r \in Z(R)^{*}$, such that $x-r-y$ is a path in $\Gamma^{*}(R)$, but is not a path in $\Gamma(R)$. Hence $C: x-r-y-x$ is a cycle in $\Gamma^{*}(R)$ of length three, which is not a cycle in $\Gamma(R)$.

Proof. Let $x$ and $y$ be distinct elements of $Z(R)^{*}$ such that $x-y$ is an edge of $\Gamma^{*}(R)$ that is not an edge of $\Gamma(R)$. Then $x y \neq 0$ and there exists $0 \neq r \in R \backslash(\operatorname{ann}(x) \cup \operatorname{ann}(y))$ such that $x r y=0$. We show that $r \notin\{x, y\}$. If $r=x$, then $x^{2} y=0$, that is a contradiction. Also if $r=y$, then $x y^{2}=0$, that is a contradiction. Thus $x-r-y$ is a path in $\Gamma^{*}(R)$, hence $x-r-y-x$ is a cycle in $\Gamma^{*}(R)$ that is not in $\Gamma(R)$. 
For a nonreduced commutative ring $R$, Badawi [3, Theorem 3.10], introduced the induced subgraph of $A G(R)$ with vertices $\operatorname{Nil}(R)^{*}$, denoted by $A G_{N}(R)$, and showed that $A G_{N}(R)$ is complete. Moreover, he defined the induced subgraph of $\Gamma(R)$ with vertices $\operatorname{Nil}(R)^{*}$, denoted by $\Gamma_{N}(R)$, and showed that $\Gamma_{N}(R)$ is complete if and only if $\operatorname{Nil}(R)^{2}=0$. Now we extend these Badawi's results to reversible rings.

Theorem 2.5. Let $R$ be a reversible ring which is not reduced with $\left|\operatorname{Nil}(R)^{*}\right| \geq 2$. If $\Gamma_{N}^{*}(R)$ is the induced subgraph of $\Gamma^{*}(R)$ with vertices $\operatorname{Nil}(R)^{*}$, then $\Gamma_{N}^{*}(R)$ is complete.

Proof. Let $a, b \in \operatorname{Nil}(R)^{*}$ be distinct elements and $m, n$ be the minimum positive integers such that $a^{m}=0$ and $b^{n}=0$. Suppose that, $a b \neq 0$. If for some positive integer $s<m, a^{s} b=0$ and $s_{0}$ is the least positive integer such that $s_{0}<m$ and $a^{s_{0}} b=0$, then we consider $r=a^{s_{0}-1}$. So $r$ is a nonzero element of $Z(R), r \notin \operatorname{ann}(a) \cup \operatorname{ann}(b)$, and $a r b=0$. Thus $a$ and $b$ are adjacent in $\Gamma_{N}^{*}(R)$. If for some positive integer $t<n, a b^{t}=0$ and $t_{0}$ is the least positive integer such that $t_{0}<n$ and $a b^{t_{0}}=0$, then we consider $r=b^{t_{0}-1}$. So $r$ is a nonzero element of $Z(R)$, $r \notin \operatorname{ann}(a) \cup \operatorname{ann}(b)$, and $a r b=0$. Thus $a$ and $b$ are adjacent in $\Gamma_{N}^{*}(R)$. If for all positive integers $s<m$ and $t<n$, we have $a^{s} b \neq 0$ and $a b^{t} \neq 0$, then we consider $r=\left(a^{m-1}+b^{n-1}\right)$, that is a nonzero element of $Z(R), r \notin \operatorname{ann}(a) \cup \operatorname{ann}(b)$, and we have $a r b=a\left(a^{m-1}+b^{n-1}\right) b=0$, thus $\Gamma_{N}^{*}(R)$ is complete.

Theorem 2.6. Let $R$ be a reversible ring which is not reduced with $\left|\operatorname{Nil}(R)^{*}\right| \geq 2$ and let $\Gamma_{N}(R)$ be the induced subgraph of $\Gamma(R)$ with vertices $\operatorname{Nil}(R)^{*}$. Then $\Gamma_{N}(R)$ is complete if and only if $\operatorname{Nil}(R)^{2}=0$.

Proof. Suppose that $\operatorname{Nil}(R)^{2}=0$. Clearly, for each pair of nonzero distinct elements of $\operatorname{Nil}(R)$, say $a$ and $b$, we have $a b=0$, and hence $\Gamma_{N}(R)$ is complete.

Conversely, suppose that $\Gamma_{N}(R)$ is complete and there exists $c \in \operatorname{Nil}(R)^{*}$, such that $c^{2} \neq 0$ and $c^{m}=0$, for some $m \in \mathbb{N}$ such that $c^{k} \neq 0$, for each $k<m$. We show that $c^{m-1}+c$ is a nonzero element. If $c^{m-1}+c=0$, then $c^{m-1}=-c$, so $c^{m-1} c=-c^{2}$, which implies that $c^{2}=0$, that is a contradiction. Moreover, $\left(c^{m-1}+c\right) c^{m-1}=0$, thus $c^{m-1}+c$ is an element of $Z(R)^{*}$. On the other hand, $c^{m-1}+c$ is a nilpotent element of $R$, since $\left(c^{m-1}+c\right)^{m}=0$. Thus $\left(c^{m-1}+c\right) \in \operatorname{Nil}(R)^{*}$. By completeness of $\Gamma_{N}(R)$, we have $c\left(c^{m-1}+c\right)=0$, but $c\left(c^{m-1}+c\right)=c^{2}$. Thus $c^{2}=0$, and hence $\operatorname{Nil}(R)^{2}=0$.

By a similar method as used in the proof of Theorem [2.6] we have the following theorem.

Theorem 2.7. Let $R$ be a nonreduced ring. Let $\left|\mathrm{Nil}(R)^{*}\right| \geq 2$ and $\Gamma_{N}(R)$ be the induced subgraph of $\Gamma(R)$ with vertices $\operatorname{Nil}(R)^{*}$. Then $\Gamma_{N}(R)$ is complete if and only if $\operatorname{Nil}(R)^{2}=0$. 
Since by Theorem [2.5, $\Gamma_{N}^{*}(R)$ is complete, so by using Theorem [2.6] we have the following corollary.

Corollary 2.8. Let $R$ be a reversible ring which is not reduced. If $\operatorname{Nil}(R)^{2} \neq 0$, then $\Gamma^{*}(R) \neq$ $\Gamma(R)$.

In the next theorem, we give a necessary condition for $\Gamma^{*}(R) \neq \Gamma(R)$.

Theorem 2.9. Let $R$ be a reversible ring. In each of the following conditions we have $\Gamma^{*}(R) \neq$ $\Gamma(R)$.

(1) If $R$ is nonreduced and $Z(R)$ is not an ideal.

(2) If there are zero-divisors $a \neq b$ such that $(0:\{a, b\})=0$ and either $(i) R$ is a reduced ring with more than two minimal primes, or (ii) $R$ is nonreduced.

Proof. By [6, Theorem 2.5], $\operatorname{diam}(\Gamma(R))=3$ and by Lemma [2.3], $\operatorname{diam}\left(\Gamma^{*}(R)\right) \leq 2$. Hence $\Gamma^{*}(R) \neq \Gamma(R)$

\section{Quasi-Zero-Divisor Graphs of Reduced Rings}

In this section, we study the diameter and girth of $\Gamma^{*}(R)$, when $R$ is reduced. We investigate, when $\Gamma^{*}(R)=\Gamma(R)$. We start this section with the following theorem. In this theorem, we study the case that $\Gamma^{*}(R) \neq \Gamma(R)$, by a similar method as used in the proof of [3, Corollary $2.5]$.

Theorem 3.1. Let $R$ be a reduced ring and $\Gamma^{*}(R) \neq \Gamma(R)$. Then $\mathrm{g}\left(\Gamma^{*}(R)\right)=3$ and there exists a cycle of length three in $\Gamma^{*}(R)$ that is not a cycle in $\Gamma(R)$.

Proof. Suppose that $x-y$ is an edge of $\Gamma^{*}(R)$ that is not an edge of $\Gamma(R)$. There exists $r \in Z(R)^{*}$ such that $r x \neq 0, r y \neq 0$ and $x r y=0$. We show that, $r \notin\{x, y\}$. If $r=x$, then $x^{2} y=0$, so $(x y)^{2}=0$, that is a contradiction. If $r=y$, then $y^{2} x=0$, so $(y x)^{2}=0$, that is a contradiction. On the other hand, $y \in R \backslash(\operatorname{ann}(x) \cup \operatorname{ann}(r))$ and $x \in R \backslash(\operatorname{ann}(y) \cup \operatorname{ann}(r))$, so $x-r-y$ is a path in $\Gamma^{*}(R)$, that is not a path in $\Gamma(R)$. Hence $C: x-r-y-x$ is a cycle of length three in $\Gamma^{*}(R)$ such that the edges of $C$ are not in $\Gamma(R)$.

In the next theorem we study the case that the completeness of $\Gamma^{*}(R)$ and $\Gamma(R)$ are equivalent. This theorem has an important role in this section.

Theorem 3.2. Let $R$ be a reduced ring. The following statements are equivalent:

(1) $\Gamma^{*}(R)$ is complete;

(2) $\Gamma(R)$ is complete (and therefore $\Gamma^{*}(R)=\Gamma(R)$ );

(3) $R$ is isomorphic to $\mathbb{Z}_{2} \times \mathbb{Z}_{2}$. 
Alg. Struc. Appl. Vol. 5 No. 2 (2018) 1-13.

Proof. (1) $\Rightarrow(2)$ Suppose that $\Gamma^{*}(R)$ is complete and $\Gamma(R)$ is not complete, thus there exist $a, b \in Z(R)^{*}$, such that $a b \neq 0$ and there exists $0 \neq r \in R \backslash(\operatorname{ann}(x) \cup \operatorname{ann}(y))$, such that $a r b=0$. If $a b=a$, then $a b r=a r=0$, that is a contradiction. Since $R$ is reduced, if $a b \in \operatorname{ann}(a)$, then $a^{2} b=0=(a b)^{2}$, so we have $a b=0$, that is a contradiction. Thus by completeness of $\Gamma^{*}(R)$, $a b$ and $a$ are adjacent, so there exists $0 \neq s \in R \backslash(\operatorname{ann}(a b) \cup \operatorname{ann}(a))$, such that $a b s a=0$. Thus $a^{2} b s=0$, which implies that $a b s=0$, a contradiction. So $a b=0$ and $\Gamma(R)$ is complete. $(2) \Rightarrow(1)$ Since every two adjacent vertices in $\Gamma(R)$ are adjacent in $\Gamma^{*}(R)$, we have the result. $(2) \Leftrightarrow(3)$ By [G, Remark 2.2], it is clear.

Theorem [3.2, has the following immediate corollary.

Corollary 3.3. Let $R$ be a reduced ring. Then $\operatorname{diam}\left(\Gamma^{*}(R)\right)=2$ if and only if $R$ is not isomorphic to $\mathbb{Z}_{2} \times \mathbb{Z}_{2}$.

Theorem 5.2 and Corollary 5.3 give a characterization for $\operatorname{diam}\left(\Gamma^{*}(R)\right)$, when $R$ is a reduced ring. The following two theorems give a necessary condition for $\Gamma^{*}(R) \neq \Gamma(R)$ and hence for $\mathrm{g}\left(\Gamma^{*}(R)\right)=3$.

Theorem 3.4. Let $R$ be a reduced ring that is not an integral domain and $Z(R)$ be an ideal of $R$. Then $\Gamma^{*}(R) \neq \Gamma(R)$ and $\mathrm{g}\left(\Gamma^{*}(R)\right)=3$.

Proof. If $\Gamma^{*}(R)$ is complete, then by Theorem $\mathbf{3 . 2}, R$ is isomorphic to $\mathbb{Z}_{2} \times \mathbb{Z}_{2}$, so $Z(R)$ is not an ideal. Thus $\Gamma^{*}(R)$ is not complete and $\Gamma^{*}(R) \neq \Gamma(R)$. So by Theorem $3.1, \mathrm{~g}\left(\Gamma^{*}(R)\right)=3$.

Theorem 3.5. Let $R$ be a reduced ring and $|\operatorname{Min}(R)| \geq 3$. Then $\Gamma^{*}(R) \neq \Gamma(R)$ and $\mathrm{g}\left(\Gamma^{*}(R)\right)=$ 3.

Proof. We have the following cases:

(a) If $\operatorname{diam}(\Gamma(R))=1$, then by $[$, $\operatorname{Remark} 2.2], R \cong \mathbb{Z}_{2} \times \mathbb{Z}_{2}$. So $|\operatorname{Min}(R)|=2$, that is a contradiction. Thus $\operatorname{diam}(\Gamma(R)) \neq 1$.

(b) If $\operatorname{diam}(\Gamma(R))=2$, then by [6, Theorem 2.5], $|\operatorname{Min}(R)|=2$. That is a contradiction.

(c) If $\operatorname{diam}(\Gamma(R))=3$, then there exist distinct elements $x, y \in Z(R)^{*}$ such that $d_{\Gamma(R)}(x, y)=3$. By Lemma [2.3, $x$ and $y$ are adjacent in $\Gamma^{*}(R)$, and hence $\Gamma^{*}(R) \neq \Gamma(R)$. So by Theorem $\mathbf{B . D}$, $\mathrm{g}\left(\Gamma^{*}(R)\right)=3$.

In the next theorem we give a necessary and sufficient condition for $\Gamma^{*}(R)=\Gamma(R)$.

Theorem 3.6. Let $R$ be a reduced ring. Then $\Gamma^{*}(R)=\Gamma(R)$ if and only if $|\operatorname{Min}(R)|=2$. 
Proof. Suppose that $\Gamma^{*}(R)=\Gamma(R)$. By Theorem [3.5, $|\operatorname{Min}(R)| \leq 2$. If $|\operatorname{Min}(R)|=1$, then $Z(R)$ is an ideal. By Theorem $[],. \Gamma^{*}(R) \neq \Gamma(R)$, that is a contradiction. Thus $|\operatorname{Min}(R)|=2$.

Converesly, assume that $R$ is reduced and $|\operatorname{Min}(\mathrm{R})|=2$. Suppose $\operatorname{Min}(R)=\left\{P_{1}, P_{2}\right\}$, so $Z(R)=P_{1} \cup P_{2}$. By [6, Theorem 2.5], $\operatorname{diam}(\Gamma(R))=2$ and by [7, Proposition 3.6], $P_{1}$ and $P_{2}$ are completely prime, $P_{1} \cap P_{2}=0$ and $P_{1} P_{2}=0=P_{2} P_{1}$. If $\Gamma^{*}(R) \neq \Gamma(R)$, then there exists distinct elements $a$ and $b$ in $Z(R)^{*}$ such that $a b \neq 0$ and for some $0 \neq r \in R \backslash(\operatorname{ann}(a) \cup \operatorname{ann}(b))$, $a r b=0=a b r$. So $a b \in P_{1}$ or $a b \in P_{2}$. Without loss of generality, assume that $a b \in P_{1}$. If $a \in P_{2}$ or $b \in P_{2}$, then $a b \in P_{2}$, a contradiction, since $P_{1} \cap P_{2}=0$. So $a \in P_{1}$ and $b \in P_{1}$. $a b r=0 \in P_{2}$ and $a b \notin P_{2}$, so $r \in P_{2}$ and hence, $r a=0=r b$, that is a contradiction. Thus $\Gamma^{*}(R)=\Gamma(R)$.

The following result give a necessary condition for $\operatorname{diam}\left(\Gamma^{*}(R)\right)=2$.

Corollary 3.7. Let $R$ be a reduced ring. If $|\operatorname{Min}(R)|=2$, then $\operatorname{diam}\left(\Gamma^{*}(R)\right)=2$.

Proof. By Theorem [3.6, $\Gamma^{*}(R)=\Gamma(R)$ and by [6, Theorem 2.5], $\operatorname{diam}(\Gamma(R))=2$. Thus $\operatorname{diam}\left(\Gamma^{*}(R)\right)=2$.

In the next theorem we give a necessary and sufficient condition for $\mathrm{g}\left(\Gamma^{*}(R)\right)=4$.

Theorem 3.8. Let $R$ be a reduced ring that is not an integral domain. The following statements are equivalent:

(1) $\mathrm{g}\left(\Gamma^{*}(R)\right)=4$;

(2) $\Gamma^{*}(R)=\Gamma(R)$ and $\mathrm{g}(\Gamma(R))=4$;

(3) $|\operatorname{Min}(R)|=2$ and each minimal prime ideal of $R$ has at least three distinct elements;

(4) $\Gamma(R)=K^{m, n}$ with $m, n \geq 2$;

(5) $\Gamma^{*}(R)=K^{m, n}$ with $m, n \geq 2$.

Proof. (1) $\Rightarrow(2)$ By Theorem [.], $\Gamma^{*}(R)=\Gamma(R)$ and thus $\mathrm{g}(\Gamma(R))=4$.

$(2) \Rightarrow(3)$ By Theorem [.6, $|\operatorname{Min}(R)|=2$. On the other hand, $\mathrm{g}(\Gamma(R))=4$, so each minimal prime ideal of $R$ has at least three distinct elements.

$(3) \Rightarrow(4)$ By [6, Theorem 2.5], $\operatorname{diam}(\Gamma(R))=2$, and by [G, Proposition 3.6], $P_{1} P_{2}=0=P_{2} P_{1}$.

We show that the nonzero elements of $P_{1}$ don't annihilate each other. If $x, y \in P_{1}$ are nonzero elements and $x y=0$, then $x y=0 \in P_{2}$, so $x \in P_{2}$ or $y \in P_{2}$, that is a contradiction. Similarly, we can show that the nonzero elements of $P_{2}$ don't annihilate each other. Thus $\Gamma(R)=K^{m, n}$, for some positive integers $m, n$. Since each minimal prime ideal of $R$ has at least three distinct elements, so $m, n \geq 2$. 
Alg. Struc. Appl. Vol. 5 No. 2 (2018) 1-13.

$(4) \Rightarrow(5)$ If $\Gamma(R)=K^{m, n}$ with $m, n \geq 2$, then $\operatorname{diam}(\Gamma(R))=2$, so by [6, Theorem 2.5], $|\operatorname{Min}(R)|=2$, thus by Theorem [.6, $\Gamma^{*}(R)=\Gamma(R)$, and hence $\Gamma^{*}(R)=K^{m, n}$ with $m, n \geq 2$. $(5) \Rightarrow(1)$ It is clear.

In the following theorem we give a necessary and sufficient condition for $g\left(\Gamma^{*}(R)\right)=\infty$.

Theorem 3.9. Let $R$ be a reduced ring that is not an integral domain. The following statements are equivalent:

(1) $\mathrm{g}\left(\Gamma^{*}(R)\right)=\infty$;

(2) $\Gamma^{*}(R)=\Gamma(R)$ and $\mathrm{g}(\Gamma(R))=\infty$;

(3) $|\operatorname{Min}(R)|=2$ and at least one minimal prime ideal of $R$ has exactly two distinct elements;

(4) $\Gamma(R)=K^{1, n}$ for some $n \geq 1$;

(5) $\Gamma^{*}(R)=K^{1, n}$ for some $n \geq 1$.

Proof. (1) $\Rightarrow(2)$ By Theorem [.], $\Gamma^{*}(R)=\Gamma(R)$ and thus $\mathrm{g}(\Gamma(R))=\infty$.

$(2) \Rightarrow(3)$ By Theorem [3.6, $|\operatorname{Min}(R)|=2$. On the other hand, $\mathrm{g}(\Gamma(R))=\infty$, so at least one minimal prime ideal of $R$ has exactly two distinct elements.

$(3) \Rightarrow(4)$ By the same argument as used in the proof of $(3) \Rightarrow(4)$ of Theorem $\mathbf{3 . 8}$, we have $\Gamma(R)=K^{m, n}$, for some positive integer $m, n$. Since at least one minimal prime ideal of $R$ has exactly two distinct elements, we have $\Gamma(R)=K^{1, n}$ with $n \geq 1$.

$(4) \Rightarrow(5)$ If $\Gamma(R)=K^{1, n}$ with $n \geq 1$, then $\operatorname{diam}(\Gamma(R))=2$, so by [6, Theorem 2.5], $|\operatorname{Min}(R)|=$ 2, thus by Theorem [3.6, $\Gamma^{*}(R)=\Gamma(R)$, so $\Gamma^{*}(R)=K^{1, n}$ with $n \geq 1$.

$(5) \Rightarrow(1)$ It is clear.

Corollary 3.10. Let $R$ be a reduced ring. Then $\Gamma^{*}(R)=\Gamma(R)$ if and only if $\mathrm{g}\left(\Gamma^{*}(R)\right)=$ $\mathrm{g}(\Gamma(R)) \in\{4, \infty\}$

\section{Quasi-Zero-Divisor Graph of $R[x]$}

Kim and Lee [9], studied extensions of reversible rings and showed that polynomial rings over reversible rings need not to be reversible in general. In this section, we study the diameter and girth of $\Gamma^{*}(R[x])$, when $R$ is reversible. We investigate, when $\Gamma^{*}(R[x])=\Gamma(R[x])$.

Definition 4.1. [5, Definition 1.1] A ring $R$ is said to be right McCoy (resp., left McCoy) if for each pair of nonzero polynomials $f(x), g(x) \in R[x]$ with $f(x) g(x)=0$ then there exists a nonzero element $r \in R$ with $f(x) r=0$ (resp., $r g(x)=0$ ). A ring is McCoy if it is both left and right McCoy. 
For a commutative ring $R$, McCoy [II], showed that a polynomial $f(x) \in R[x]$ is a zerodivisor if and only if there is a nonzero element $r \in R$ such that $r f(x)=0$. Afterward, Nielsen [12, Theorem 2], showed that reversible rings are McCoy. We use this property of reversible rings. In the following Lemma, we show that for a reversible ring $R, \operatorname{diam}\left(\Gamma^{*}(R[x])\right) \leq 2$.

Lemma 4.2. Let $R$ be a reversible ring. Let $f(x)$ and $g(x)$ be distinct elements of $Z(R[x])^{*}$. If $d_{\Gamma(R[x])}(f(x), g(x))=3$, then $d_{\Gamma^{*}(R[x])}(f(x), g(x))=1$ and $\operatorname{diam}\left(\Gamma^{*}(R[x])\right) \leq 2$.

Proof. Let $f(x)$ and $g(x)$ be distinct elements of $Z(R[x])^{*}$. Since $R$ is McCoy, there exists $a$ and $b$ in $R \backslash\{0\}$, such that $f(x) a=0$ and $b g(x)=0$. On the other hand, $(a+b) \neq 0$, $a g(x) \neq 0 \neq g(x) a$ and $b f(x) \neq 0 \neq f(x) b$, since $f(x)$ and $g(x)$ have no mutual annihilator. So $(a+b)$ is a nonzero element of $R$. Note that, $(a+b) f(x)=b f(x) \neq 0, f(x)(a+b)=$ $f(x) b \neq 0,(a+b) g(x)=a g(x) \neq 0$, and $g(x)(a+b)=g(x) a \neq 0$. We set $r=a+b$, then $0 \neq r \in R[x] \backslash(\operatorname{ann}(f(x)) \cup \operatorname{ann}(g(x)))$, and $f(x) r g(x)=0$. Thus $d_{\Gamma^{*}(R[x]}(f(x), g(x))=1$.

Lemma 4.3. Let $R$ be a reversible ring and $f(x)-g(x)$ is not an edge of $\Gamma^{*}(R[x])$. Then there exists $h(x) \in Z(R[x])^{*} \backslash\{f(x), g(x)\}$ such that, $f(x)-h(x)-g(x)$ is a path in $\Gamma(R[x])$, and hence $f(x)-h(x)-g(x)$ is a path in $\Gamma^{*}(R[x])$.

Proof. Let $f(x)$ and $g(x)$ be distinct elements of $Z(R[x])^{*}$. If $f(x)$ and $g(x)$ are not adjacent in $\Gamma^{*}(R[x])$, then $f(x)$ and $g(x)$ are not adjacent in $\Gamma(R[x])$. Hence $f(x) g(x) \neq 0$ and $g(x) f(x) \neq 0$. On the other hand, $\operatorname{diam}(\Gamma(R[x])) \leq 3$. If $d_{\Gamma(R[x])}(f(x), g(x))=3$, then $d_{\Gamma^{*}(R[x])}(f(x), g(x))=1$, that is a contradiction. Thus $d_{\Gamma(R[x])}(f(x), g(x))=2$. So there exists $h(x) \in Z^{*}(R[x]) \backslash\{f(x), g(x)\}$ which is a nonzero mutual annihilator of $f(x)$ and $g(x)$. Hence $f(x)-h(x)-g(x)$ is a path in $\Gamma(R[x])$.

Theorem 4.4. Let $R$ be a reversible ring which is not reduced. If $\Gamma_{N}^{*}(R[x])$ is induced subgraph of $\Gamma^{*}(R[x])$ with vertices $\operatorname{Nil}(R[x])^{*}$, then $\Gamma_{N}^{*}(R[x])$ is complete.

Proof. Let $f(x)$ and $g(x)$ be distinct elements of $\operatorname{Nil}(R[x])^{*}$. Then there exist $n, m \in \mathbb{N}$ such that $f^{n}(x)=0$ and $g^{m}(x)=0$. Suppose that $n$ be the least positive integer such that $f^{n}(x)=0$ and $m$ be the least positive integer such that $g^{m}(x)=0$. If $f(x) g(x)=0$ or $g(x) f(x)=0$, then we have the result. Otherwise, we have at least one of the following cases :

(i) Suppose that for some positive integer $k<n, f^{k}(x) g(x)=0$ and for some positive integer $\ell<n, g(x) f^{\ell}(x)=0$, and $k$ is the least positive integer such that $f^{k}(x) g(x)=0$, and $\ell$ is the least positive integer such that $g(x) f^{\ell}(x)=0$. If $\min \{k, \ell\}=\ell$, then we set $h(x)=f^{\ell-1}(x)$, thus $0 \neq h(x) \in R[x] \backslash(\operatorname{ann}(f(x)) \cup \operatorname{ann}(g(x)))$ and $g(x) h(x) f(x)=0$. If $\min \{k, \ell\}=k$, then we set $h(x)=f^{k-1}(x)$, thus $0 \neq h(x) \in R[x] \backslash(\operatorname{ann}(f(x)) \cup \operatorname{ann}(g(x)))$ and $f(x) h(x) g(x)=0$. 
(ii) Suppose that for some positive integer $k<m, g^{k}(x) f(x)=0$ and for some positive integer $\ell<m, f(x) g^{\ell}(x)=0$, and $k$ is the least positive integer such that $g^{k}(x) f(x)=0$, and $\ell$ is the least positive integer such that $f(x) g^{\ell}(x)=0$. If $\min \{k, \ell\}=\ell$, then we set $h(x)=g^{\ell-1}(x)$, thus $0 \neq h(x) \in R[x] \backslash(\operatorname{ann}(f(x)) \cup \operatorname{ann}(g(x)))$ and $f(x) h(x) g(x)=0$. If $\min \{k, \ell\}=k$, then we set $h(x)=g^{k-1}(x)$, thus $0 \neq h(x) \in R[x] \backslash(\operatorname{ann}(f(x)) \cup \operatorname{ann}(g(x)))$ and $g(x) h(x) f(x)=0$.

(iii) If for some positive integer $k<n, f^{k}(x) g(x)=0$ and for each positive integer $\ell<n$, $g(x) f^{\ell}(x) \neq 0$, and $k$ is the least positive integer such that $f^{k}(x) g(x)=0$, then we set $h(x)=f^{k-1}(x)$, thus $0 \neq h(x) \in R[x] \backslash(\operatorname{ann}(f(x)) \cup \operatorname{ann}(g(x)))$, and $f(x) h(x) g(x)=0$. (iv) If for some positive integer $\ell<n, g(x) f^{\ell}(x)=0$ and for each positive integer $k<n$, $f^{k}(x) g(x) \neq 0$, and $\ell$ is the least positive integer such that $g(x) f^{\ell}(x)=0$, then we set $h(x)=f^{\ell-1}(x)$, thus $0 \neq h(x) \in R[x] \backslash(\operatorname{ann}(f(x)) \cup \operatorname{ann}(g(x)))$, and $g(x) h(x) f(x)=0$.

$(v)$ If for some positive integer $k<m, f(x) g^{k}(x)=0$ and for each positive integer $\ell<m$, $g^{\ell}(x) f(x) \neq 0$, then by the similar argument as used in case $(i i i)$, we have the result.

(vi) If for some positive integer $\ell<m, g^{\ell}(x) f(x)=0$ and for each positive integer $k<m$, $f(x) g^{k}(x) \neq 0$, then by the similar argument as used in case $(i v)$, we have the result.

(vii) If for each $s<m, f(x) g^{s}(x) \neq 0 \neq g^{s}(x) f(x)$, and for each $t<n$, $g(x) f^{t}(x) \neq 0 \neq f^{t}(x) g(x)$, then we set $h(x)=f^{n-1}(x)+g^{m-1}(x)$. We show that $h(x) \neq 0$. If $h(x)=0$, then $f^{n-1}(x)=-g^{m-1}(x)$, so $0=f(x) f^{n-1}(x)=-f(x) g^{m-1}(x)$, that is a contradiction. Thus $0 \neq h(x) \in R[x] \backslash(\operatorname{ann}(f(x)) \cup \operatorname{ann}(g(x)))$ and $f(x) h(x) g(x)=0$.

Thus $\Gamma_{N}^{*}(R[x])$ is complete.

Since by using Theorem $4.4, \Gamma_{N}^{*}(R[x])$ is complete, whenever $R$ is a reversible ring which is not reduced, so by using Theorem [2.], we have the following proposition.

Proposition 4.5. Let $R$ be a reversible ring which is not reduced. If $\operatorname{Nil}(R[x])^{2} \neq 0$, then $\Gamma^{*}(R[x]) \neq \Gamma(R[x])$.

C.Y. Hong, N.K. Kim, Y. Lee and S.J. Ryu in [8, Definition 1.1], introduced the right (resp., left) Property (A) of a ring $R$. We say that a ring $R$ has right ( resp., left) Property $(A)$ if for every finitely generated two-sided ideal $I \subseteq Z_{\ell}(R)$ (resp., $Z_{r}(R)$ ), there exists nonzero $a \in R$ (resp., $b \in R$ ) such that $I a=0$ (resp., $b I=0$ ). A ring $R$ is said to have Property $(A)$ if $R$ has right and left $\operatorname{Property}(A)$. In the following theorem we give a necessary condition for $\Gamma^{*}(R[x]) \neq \Gamma(R[x])$.

Theorem 4.6. Let $R$ be a reversible ring. If $R$ is not a reduced ring with exactly two minimal primes and either $R$ has not $\operatorname{Property}(A)$ or $Z(R)$ is not an ideal, then $\Gamma^{*}(R[x]) \neq \Gamma(R[x])$. 
Proof. By [6, Theorem 2.7], $\operatorname{diam}(\Gamma(R[x]))=3$, and by Lemma 4.2, $\operatorname{diam}\left(\Gamma^{*}(R[x])\right) \leq 2$. Hence $\Gamma^{*}(R[x]) \neq \Gamma(R[x])$.

Proposition 4.7. Let $R$ be a reversible ring which is not reduced. Then $g\left(\Gamma^{*}(R[x])\right)=3$.

Proof. Let $a \in \operatorname{Nil}(R)^{*}$ and $m$ be the minimum positive integer such that $a^{m}=0$. We consider $r=a^{m-2}$, which is not an element of $\operatorname{ann}(a)$, and $a r a=a^{m}=0$. So, $a-a x-a x^{2}-a$ is a cycle in $\Gamma^{*}(R[x])$.

Theorem 4.8. Let $R$ be an associative ring with identity. Then $\operatorname{diam}\left(\Gamma^{*}(R[x])\right) \geq 1$, and $\mathrm{g}\left(\Gamma^{*}(R[x])\right) \in\{3,4\}$.

Proof. Let $a$ be a nonzero zero-divisor of $R$. Then $a b=0$ or $b a=0$, for some $0 \neq b \in R$. Then $a x \neq b x^{2}$ and $(a x)\left(b x^{2}\right)=0$ or $\left(b x^{2}\right)(a x)=0$, thus $d_{\Gamma^{*}(R[x])}\left(a x, b x^{2}\right)=1$, which implies that $\operatorname{diam}\left(\Gamma^{*}(R[x])\right) \geq 1$. On the other hand, $a-b x-a x^{2}-b x^{3}-a$, is a cycle of lenght four in $\Gamma^{*}(R[x])$. If $R$ is nonreduced, by Proposition $4.7, \mathrm{~g}\left(\Gamma^{*}(R[x])\right)=3$.

\section{ACKNowledgments}

The authors wish to sincerely thank the referees for several useful comments.

\section{REFERENCES}

[1] D.F. Anderson and P.S. Livingston, The zero-divisor graph of a commutative ring, J. Algebra 217 (1999) 434-447.

[2] D.F. Anderson and S.B. Mulay, On the diameter and girh of a zero-divisor graph, J. Pure Appl. Algebra 210 (2007) 543-550.

[3] A. Badawi, On the annihilator graph of a commutative ring, Commun. Algebra 42 (2014) 1-14.

[4] I. Beck, Coloring of commutative rings, J. Algebra 116 (1988) 208-226.

[5] V. Camillo and P.P. Nielsen, McCoy rings and zero-divisors, J. Pure Appl. Algebra 212 (2008) 599-615.

[6] E. Hashemi, R. Amirjan and A. Alhevaz, On zero-divisor graphs of skew polynomial rings over noncommutative rings, J. Algebra Appl. 16 (2017) 1750056 (14 pages).

[7] E. Hashemi and R. Amirjan, Zero-divisor graphs of Ore extensions over reversible rings, Canad. Math. Bull. 59 (2016) 794-805

[8] C.Y. Hong, N.K. Kim, Y. Lee and S.J. Ryu, Rings with Property (A) and their extensions, J. Algebra 315 (2007) 612-628

[9] N.K. Kim and Y. Lee, Extention of reversible rings, J. Pure Appl. Algebra 185 (2003) 207-223.

[10] T.Y. Lam, A First Course in Noncommutative Rings, Springer-Verlag, Berlin-Heidelberg-New York, 1991.

[11] N.H. McCoy, Remarks on divisors of zero, Amer. Math. Monthly 49 (1942) 286-295.

[12] P.P. Nielsen, Semi-commutativity and the McCoy condition, J. Algebra 298 (2006) 134-141. 
Alg. Struc. Appl. Vol. 5 No. 2 (2018) 1-13.

[13] S.P. Redmond, The zero-divisor graph of a non-commutative ring, Int. J. Commut. Rings 1 (2002) $203-211$.

[14] D.B. West, Introduction to Graph Theory, Second Edition, Prentice Hall, Upper Saddle River, 2001.

[15] S. Zhao, J. Nan and G. Tang, Quasi-zero-divisor graphs of non-commutative rings, J. Math. Res. Appl. 37 (2017) 137-147.

\section{Raziyeh Amirjan}

Faculty of Mathematical sciences,

Shahrood University of Technology,

Shahrood, Iran.

raziyehamirjan@gmail.com

\section{Ebrahim Hashemi}

Faculty of Mathematical sciences,

Shahrood University of Technology,

Shahrood, Iran.

eb_ hashemi@yahoo.com 